\title{
Factors Affecting the Compression and Energy Absorption Properties of Small-Sized Thin-Walled Metal Tubes
}

\author{
Xiaoqin Hao $\mathbb{D},{ }^{1,2}$ Jia $\mathrm{Yu}^{2}$ Weidong $\mathrm{He}^{2}$ and Yi Jiang $\mathbb{D}^{1}$ \\ ${ }^{1}$ Beijing Institute of Technology, Beijing 100081, China \\ ${ }^{2}$ Beijing Institute of Specialized Machinery, Beijing 100143, China \\ Correspondence should be addressed to Xiaoqin Hao; twhey2@126.com
}

Received 16 September 2019; Revised 12 December 2019; Accepted 17 January 2020; Published 15 February 2020

Academic Editor: Rosario Pecora

Copyright (C) 2020 Xiaoqin Hao et al. This is an open access article distributed under the Creative Commons Attribution License, which permits unrestricted use, distribution, and reproduction in any medium, provided the original work is properly cited.

To solve the problem of the effective cushioning of fast-moving mechanical components in small ring-shaped spaces, the factors affecting the compression and energy absorption properties of small-sized hollow metal tubes were studied. Simulation models were constructed to analyse the influences of tube diameter, wall thickness, relative position, and number of stacked components on the compression and energy absorption properties. The correctness of the simulation method and its output were verified by experiments, which proved the effectiveness of compression and energy absorption properties of small-sized thin-walled metal tubes. The research provides support for the application of metal tube buffers in armament launch technology and engineering practice.

\section{Introduction}

With the development of engineering science and technology, the problem of buffer energy absorption caused by mechanical collision in industry has become more prominent. Since the 1970s, attention has been paid to research and development into energy-absorbing structures and materials that dissipate kinetic energy from collisions (or from strong dynamic loads), especially in the automotive and military industries [1]. In recent years, the problem of buffer energy absorption within a narrow space has arisen as an important topic of interest in the aerospace industry. With the development of material science, various types of buffer energy-absorbing materials have been put into use, which has stimulated the creation of different types of buffer energy-absorbing devices. At present, the composite devices with good performance and a wide range of application include metal tube structures, aluminium honeycomb, foamed metal materials, and porous materials used to infill thin-walled structures. These buffer energy absorption devices are all based on the principle of irreversible deformation of materials and absorption of energy through plastic deformation.
The common use of thin-walled components as energyabsorbing devices is due to many important aspects including superior performance under dynamic loading, cost-effectiveness, high efficiency, and ease of manufacturing and installation [2]. Thin-walled energy absorbers have been used in many applications including aircraft subfloor structures [3], front structures of cars and trains [4], and rollover protective structures (ROPS) on items of heavy equipment used in agriculture and construction, such as earthmoving machinery and tractors [5].

The principal factors that affect the energy absorption capability of such thin-walled components are materials, structural geometry, and load regime. The most commonly used materials for manufacturing a thin-walled energy absorber are metallic, such as aluminium alloy and mild steel, or fibre-reinforced composites (FRCs). The energy dissipating mechanisms of metallic and composite structures are considerably different. The structures made from composite materials are normally brittle and dissipate energy through different combined fracture mechanisms such as delamination, fibre breakage, and matrix cracking [6], whereas the ductility of metallic structures allows them to dissipate energy through progressive plastic deformation $[1,6]$. For 
metallic tubes, both square [7] and circular cross sections have been studied before.

The influence factors and behaviour of the buffering energy absorption characteristics of small-sized thin-walled metal tubes were studied in the present research. These kinds of metal tubes are very different from those described above, as they are installed within annular openings with a diameter of 6 to $8 \mathrm{~mm}$; therefore, the diameter of the metal tube is less than $6 \mathrm{~mm}$, and its thickness is 0.8 to $1.2 \mathrm{~mm}$. The metal tubes are annular and are stacked along the axial direction.

According to the early research, the axial crushing of circular tubes was extensively studied by many researchers [8-11] interested in their energy absorption capability. Those authors were among the first researchers to develop an analytical model for the axial collapse of a circular tube. The axial crushing of a circular tube involves progressive folding or buckling of the tube with one or more of three main deformation modes: axisymmetric or ring, nonsymmetric or diamond, or a mixed mode [2]. A novel method to improve energy absorption performance of thin-walled circular tubes under quasistatic lateral loading through a preforming process was also investigated [12].

A novel solution was established, and the study of the influencing factors and rules of behaviour can be used as a reference to design buffering devices in the narrow space of high-speed mechanical moving parts and provides support for the application of metal tube-type buffering devices in weapon launching technology and aerospace engineering.

\section{Theory and Model}

2.1. Geometric Model. The geometry model is shown in Figure 1. Under external force, the descending object moves relative to the ascending object at a certain speed to simulate the collision environment. As a result, the metal tube sandwiched between them is compressed and deformed.

All the materials used in this study were designed by the authors and were manufactured by a collaborating industrial partner.

2.2. Material Model. When the changing load acts on the structure at a high speed, it deforms rapidly and the strain rate is higher. A large number of studies have shown that the mechanical properties of materials under a high strain rate are quite different from those governing quasistatic deformation, and the yield limit of materials increases with the strain rate. Among the strain rate-related constitutive relations of various engineering materials, the CowperSymonds relationship [13] is the most widely used constitutive model in structural collision simulation. This relation represents an ideal rigid-plastic material whose dynamic yield limit is related to the strain rate.

$$
\frac{\sigma_{s}^{d}}{\sigma_{s}}=1+\left(\frac{\dot{\varepsilon}}{B}\right)^{1 / q}
$$

where $\sigma_{s}^{d}$ and $\sigma_{s}$, respectively, represent the dynamic yield limit and quasistatic yield limit of the material, $\dot{\varepsilon}$ represents the strain rate of the material, and $B$ and $q$ are material

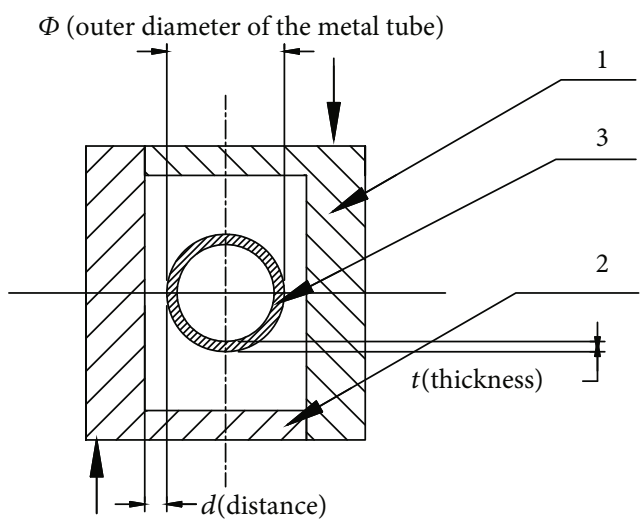

Figure 1: Compression model for a hollow metal tube. (1) Descending object. (2) Ascending object. (3) Metal tube to be compressed. $\Phi$ : outer diameter of the metal tube; $t$ : thickness of the metal tube; $d$ : distance from the outer wall of the metal tube to the wall of the inner moving object.

parameters used to characterise the rate-dependent sensitivity of the material.

In the process of structural collision, the energy absorbed in the elastic stage is negligible and a large amount of energy is dissipated in the plastic deformation stage. Therefore, the buffer material can be approximately abstracted as a rigidplastic material, and the higher the yield limit, the more energy absorbed by the material. The moving part is made of steel Q345, and the thin-walled tube is made of stainless steel $1 \mathrm{Cr} 18 \mathrm{Ni}$ Tii. The material parameters are summarised in Table 1 (all parameters were measured using an MTS testbed in the laboratory).

2.3. Compression Contact Model. A Hertzian-damped model $[14,15]$ was selected in the study of the compression of metal tubes herein, and a contact unit was introduced to simulate the contact behaviour. The two planes make contact with, and squeeze, the metal tube. The model contains a nonlinear spring and a nonlinear damper to simulate the nonlinearity and energy dissipation of contact stiffness. The collision load can be expressed as

$$
F_{c}= \begin{cases}k_{h} \delta^{3 / 2}+c_{h} \dot{\delta}, & \delta>0, \\ 0, & \delta \leq 0,\end{cases}
$$

where $k_{h}$ is the contact stiffness, $\delta$ is the relative deformation of two objects at the contact point, and $\dot{\delta}$ is the relative deformation velocity of two objects at the contact point. $c_{h}$ represents the damping coefficient, which can be expressed as

$$
c_{h}=\lambda \delta^{3 / 2}
$$

where $\lambda$ is called the hysteresis damping coefficient, which can be expressed as

$$
\lambda=\frac{3 k_{h}\left(1-e^{2}\right)}{4 \Delta u},
$$


TABLE 1: Material parameters.

\begin{tabular}{lcccc}
\hline Material & Young's modulus $(E)$ & Poisson ratio $(v)$ & Density $(\rho)$ & Tangent modulus $(G)$ \\
\hline Q345 & $201 \mathrm{GPa}$ & 0.3 & $7800 \mathrm{~kg} / \mathrm{m}^{3}$ & $1.17 \mathrm{GPa}$ \\
1 Cr18Ni9Ti & $184 \mathrm{GPa}$ & 0.3 & $7900 \mathrm{~kg} / \mathrm{m}^{3}$ & $4.13 \mathrm{GPa}$ \\
\hline
\end{tabular}

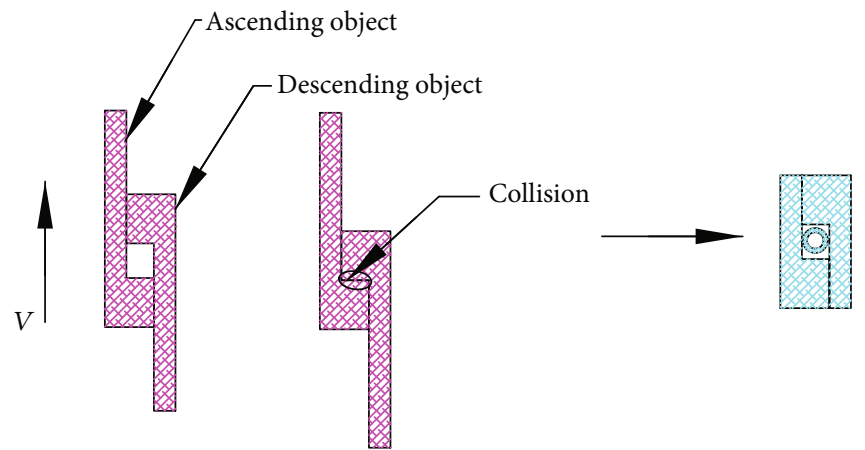

Figure 2: Finite element model.

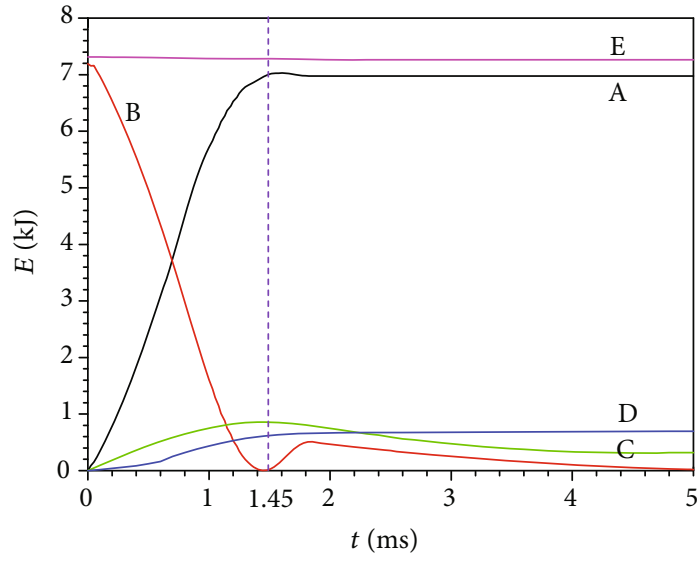

(a) $6 \mathrm{~mm}$

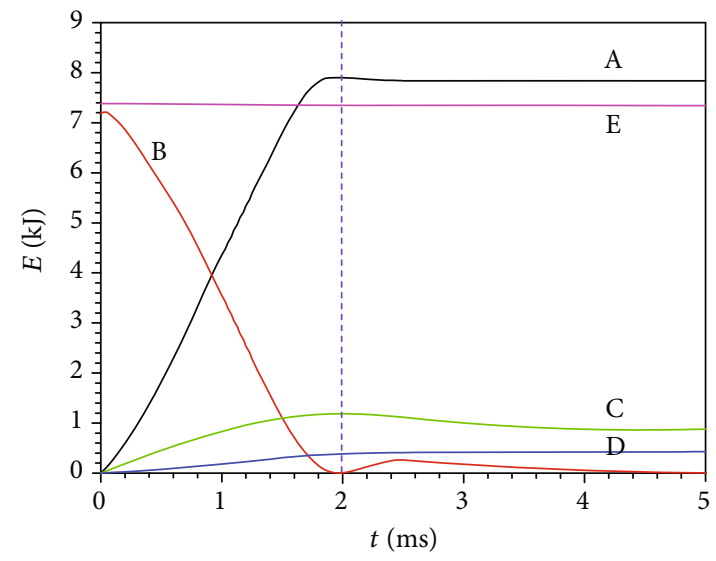

(b) $5 \mathrm{~mm}$

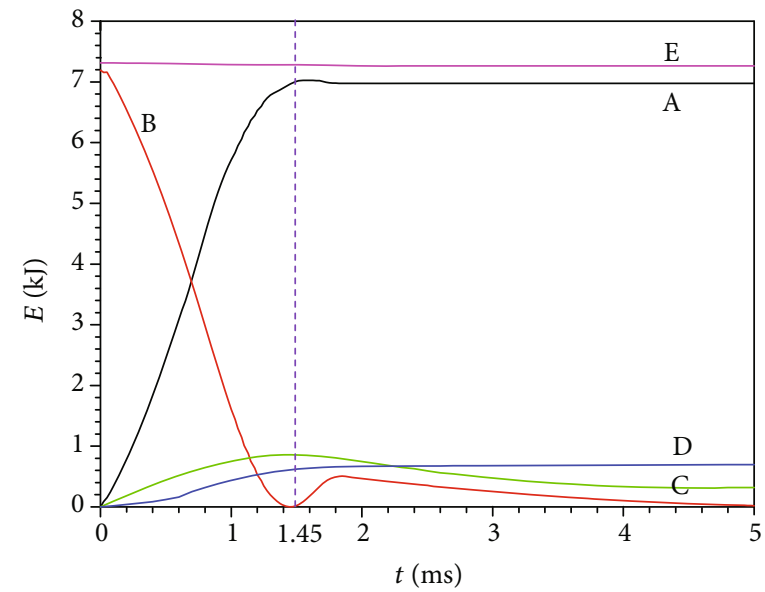

(c) $4 \mathrm{~mm}$

FIGURE 3: Energy absorption process for metal tubes of different outer diameters. A: internal energy of the metal tube; B: kinetic energy of the ascending object; C: work done by external load; D: frictional energy consumption; E: total energy of the model. 


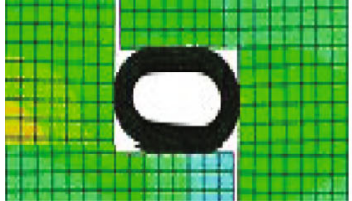

(a)

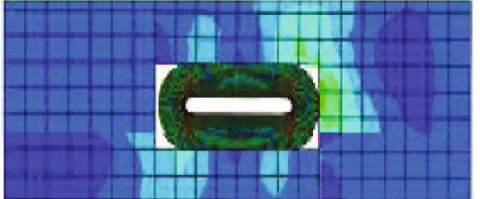

(b)

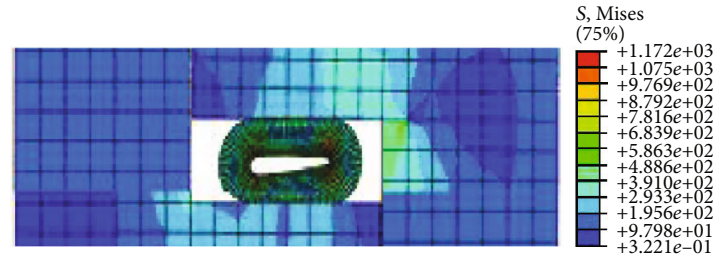

(c)

FIGURE 4: Compression equivalent stress for metal tubes of different outer diameters.

where $\Delta u$ is the velocity of the collision contact point at the moment of collision and $e$ is the energy recovery coefficient, which is used to represent the energy loss in the collision process: $e=1$ represents a completely elastic collision with no energy loss and $e=0$ represents a completely inelastic collision with the greatest energy loss.

The energy recovery coefficient is expressed in

$$
e=\frac{\Delta E_{T}-\Phi^{a}}{\Delta E_{T}+\Phi^{a}}
$$

where $\Phi^{a}$ is the energy lost in the collision compression process and $\Delta E_{T}$ is the loss of kinetic energy before and after the collision.

The contact stiffness is given by

$$
k_{h}=\frac{4}{3} E^{*} R^{* 1 / 2},
$$

where $E^{*}$ is the equivalent elastic modulus and $R^{*}$ is the equivalent radius.

The dynamic equation of compression collision is given by

$$
\mathbf{M} \ddot{\mathbf{u}}+\mathbf{C} \dot{\mathbf{u}}+\mathbf{K u}=\mathbf{F}(\mathbf{u}),
$$

where $\mathbf{M}, \mathbf{C}$, and $\mathbf{K}$, respectively, represent the mass matrix, damping matrix, and stiffness matrix, $\mathbf{u}$ is the displacement vector, and $\mathbf{F}$ is the external load vector. Except to satisfy the dynamic equation, the condition of the contact boundary must be met. The normal contact boundary condition can be discretised into

$$
\mathbf{B}_{n} \mathbf{u}+\mathbf{D}_{0} \geq 0
$$

where $\mathbf{B}_{n}$ is the normal contact constraint matrix and $\mathbf{D}_{0}$ represents the initial normal gap. The tangential contact boundary conditions can be discretised into

$$
\mathbf{B}_{\tau}\left(\mathbf{u}^{t+\Delta t}-\mathbf{u}^{t}\right)=0,
$$

where $\mathbf{B}_{\tau}$ represents the tangential contact constraint matrix and $\mathbf{u}^{t}$ and $\mathbf{u}^{t+\Delta t}$ represent displacement vectors at $t$ and $t+\Delta t$, respectively. The use of the Lagrange multiplier method, penalty function method, and linear programming is commonplace when introducing the contact condition into the dynamic equation. Here, a penalty

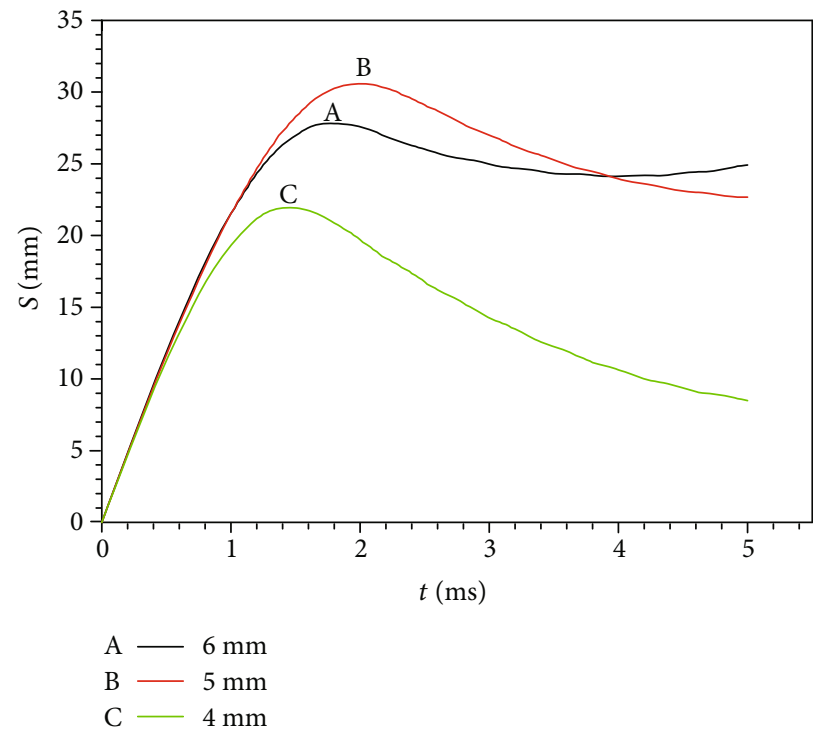

FIgURE 5: Comparison of displacement-time curves of the moving object for metal tubes of different outer diameters.

function method is adopted, which involves solving the extremum of the functional [16-18], namely:

$$
\prod(\mathbf{u})=E+\int_{t}^{t+\Delta t} \int_{S_{c}} \alpha\left(\mathbf{B}_{n} \mathbf{u}+\mathbf{D}_{0}\right)^{T}\left(\mathbf{B}_{n} \mathbf{u}+\mathbf{D}_{0}\right) d S d t
$$

where $E$ represents the total potential energy of the system, represents the contact boundary, and $\alpha$ represents the penalty function factor.

Through the above analysis, equations (2), (5), (6), (7), and (10) constitute complete kinetic equations of motion in a collision. By introducing an appropriate material constitutive model and finite element discrete method, the whole collision compression contact process can be analysed.

2.4. Finite Element Model. The finite element model is shown in Figure 2. The load and boundary conditions of moving parts are axisymmetric, so a two-dimensional axisymmetric model is used to reduce the calculation time in the ABAQUS software suite adopted here. Four-noded quadrilateral axisymmetric linear reduced integration units $(\mathrm{CAx} 4 \mathrm{R})$ are used in the mesh, and hourglass control is adopted. The mesh size of ascending and descending parts is $0.8 \mathrm{~mm}$, and the mesh size of the metal tube is $0.1 \mathrm{~mm}$. A predefined vertical upward 
TABLE 2: Simulation results: compression and energy absorption process parameters for metal tubes of different diameters.

\begin{tabular}{|c|c|c|c|}
\hline Outer diameter of the metal tube (mm) & 6 & 5 & 4 \\
\hline Time of compression and energy absorption (ms) & 1.75 & 2 & 1.45 \\
\hline Biggest equivalent stress of the moving part ( $\mathrm{MPa})$ & 840 & 440 & 550 \\
\hline Energy absorption ratio (\%) & 59.4 & 93.5 & 87 \\
\hline
\end{tabular}

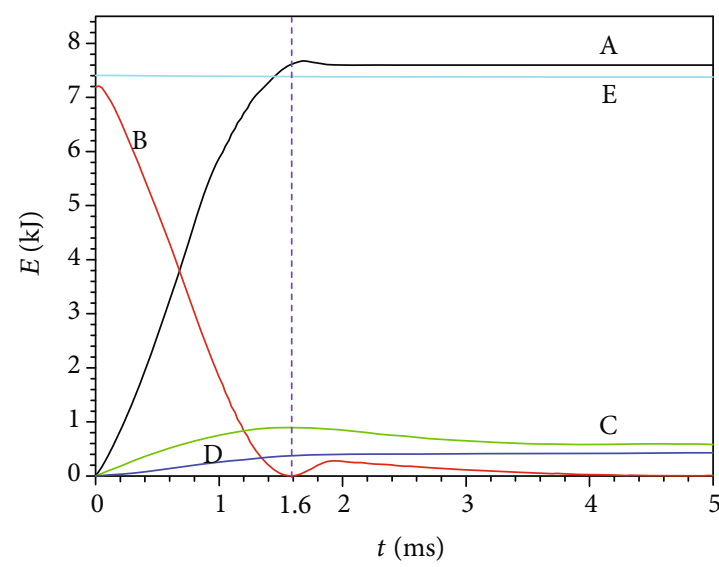

(a) $1.2 \mathrm{~mm}$

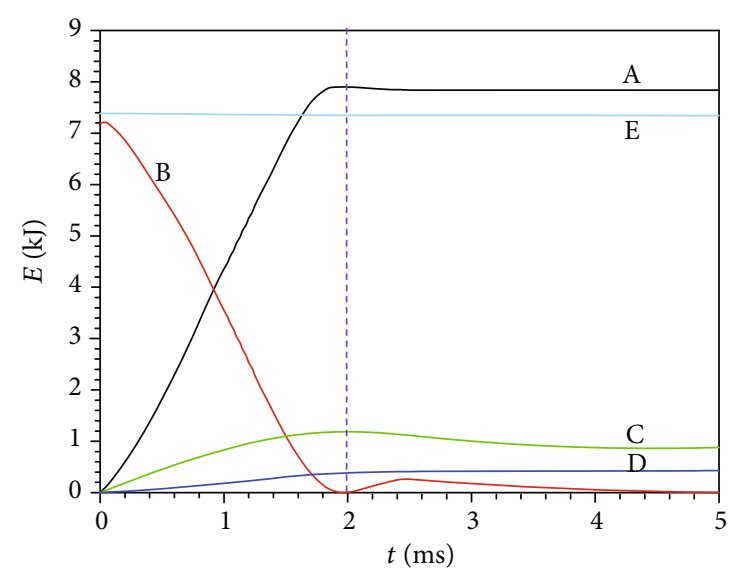

(b) $1.0 \mathrm{~mm}$

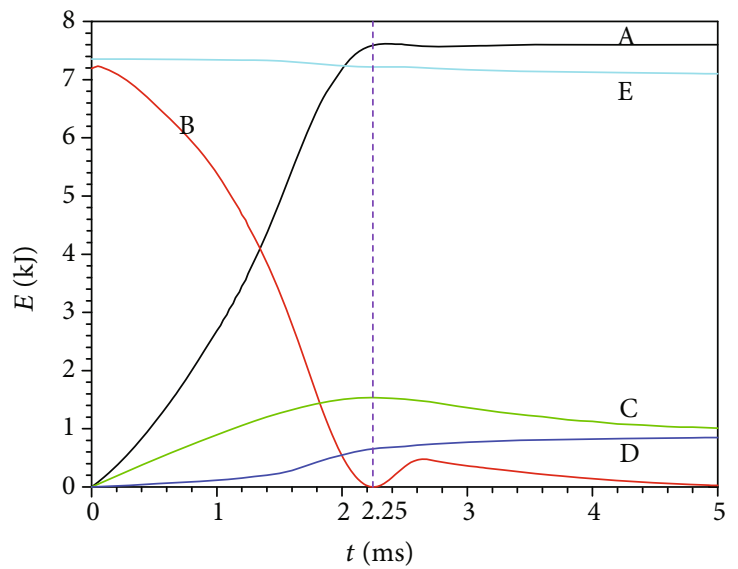

(c) $0.8 \mathrm{~mm}$

FIGURE 6: Energy conversion curves in the compression and energy absorption process of metal tubes of different wall thicknesses. A: internal energy of the metal tube; B: kinetic energy of the ascending object; C: work done under external load; D: frictional energy consumption; E: total energy of the model.

velocity field is applied to the moving parts, which can provide the initial kinetic energy for the moving part in the collision.

\section{Simulation Calculation}

3.1. Influence of Outer Diameter Size of a Metal Tube on Compression Energy Absorption Performance. A metal tube with a wall thickness of $1 \mathrm{~mm}$ is taken as the research object, and three working conditions with outer diameters of $4 \mathrm{~mm}$, $5 \mathrm{~mm}$, and $6 \mathrm{~mm}$ were taken as the research objects.

3.1.1. Comparison of Energy Conversion Performance. The energy changes in the simulation process of each working condition are shown in Figure 3. According to the curve in the figure, the energy absorption rate of the metal tube is $59.4 \%$ at $6 \mathrm{~mm}$. At $5 \mathrm{~mm}$, the energy absorption rate of the metal tube reaches $93.5 \%$. The energy absorption rate of the metal tube is $87 \%$ at $4 \mathrm{~mm}$; therefore, the energy absorption effect of $5 \mathrm{~mm}$ thin-walled rings in this model is better than that in the other two working conditions.

3.1.2. Comparison of Structural Stress Distribution. The equivalent stress distribution of moving parts under different working conditions is shown in Figure 4. From the figure, at $6 \mathrm{~mm}$, the compression of the metal tube is not large and the stress caused by extrusion on the wall of the moving part can reach a maximum of $840 \mathrm{MPa}$. At $4 \mathrm{~mm}$, the maximum stress on the moving parts can reach $550 \mathrm{MPa}$. At $5 \mathrm{~mm}$, the 


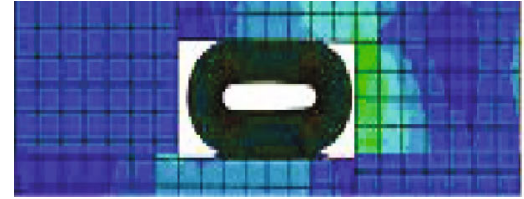

(a)

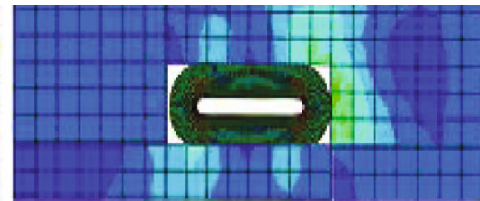

(b)

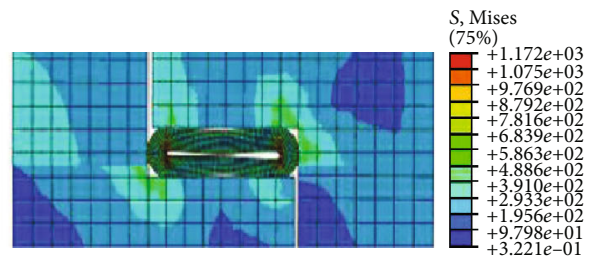

(c)

Figure 7: Compression of metal tubes of different wall thicknesses (distribution cloud picture of equivalent stress).

maximum stress on the moving parts can reach $440 \mathrm{MPa}$ and the buffering effect is good.

\subsubsection{Comparison of Deceleration Performance of Moving} Parts. The three displacement-time curves in Figure 5 show the motion of moving parts under the action of metal tubes with different outer diameters. The three curves reach their highest points at three points $\mathrm{A}, \mathrm{B}$, and $\mathrm{C}$, respectively. This indicates that the outer diameter of the metal tube is not proportional to the compression time and compression stroke. Among them, when the outer diameter of the metal tube is $5 \mathrm{~mm}$, it can be compressed with the longest buffer time and compression stroke.

3.1.4. Summary of Results. The performance parameters of the three kinds of metal tubes with different diameters after compression are summarised in Table 2 . The analysis shows that the compression energy absorption effect of the thinwalled tube with an outer diameter of $5 \mathrm{~mm}$ is better than those of $6 \mathrm{~mm}$ and $4 \mathrm{~mm}$. Therefore, the metal tube with an outer diameter of $5 \mathrm{~mm}$ is selected for subsequent analysis.

3.2. Influence of the Wall Thickness of Metal Tubes on Compression and Energy Absorption Performance. The metal tube with the outer diameter of $5 \mathrm{~mm}$ is taken as the research object. Three working conditions (wall thicknesses of $1.2 \mathrm{~mm}, 1.0 \mathrm{~mm}$, and $0.8 \mathrm{~mm}$ ) were studied to compare the effect of different wall thicknesses on buffer performance.

\subsubsection{Comparison of Energy Conversion Performance. Energy} conversion in the three working conditions is shown in Figure 6. At $1.2 \mathrm{~mm}$, the energy absorption rate of the metal tube is $93.8 \%$. At $1.0 \mathrm{~mm}$, the energy absorption rate of the metal tube can reach $93.5 \%$. At $0.8 \mathrm{~mm}$, the energy absorption rate of the metal tube is $87 \%$. This shows that the energy absorption rate of the metal tube with a thickness of $0.8 \mathrm{~mm}$ is lower, and there is little difference between the energy absorption rates of the metal tube with a thickness of $1.0 \mathrm{~mm}$ and $1.2 \mathrm{~mm}$.

3.2.2. Comparison of Structural Stress Distribution. The effective stress distribution of the three working conditions is shown in Figure 7. The compression of the metal tube with a thickness of $1.2 \mathrm{~mm}$ is smaller than that of one of $1.0 \mathrm{~mm}$, and the compression of the metal tube with a thickness of $0.8 \mathrm{~mm}$ is the largest. Therefore, the transverse size is larger after compression. At $1.2 \mathrm{~mm}$, the maximum equivalent stress on the side wall of moving parts is $480 \mathrm{MPa}$; at $1.0 \mathrm{~mm}$, the maximum equivalent stress on the side wall of

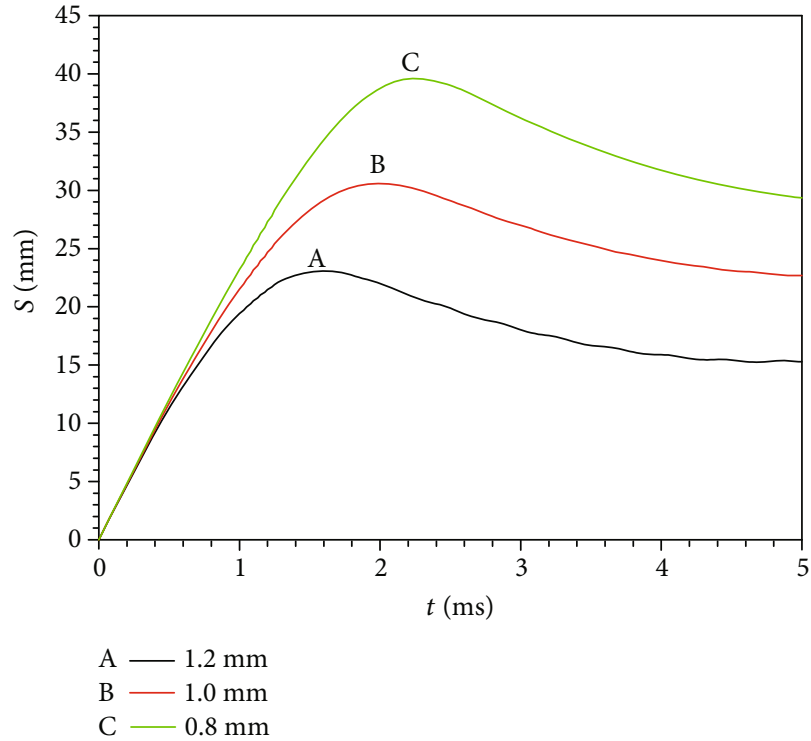

FIgURE 8: Comparison of displacement-time curves of the ascending object for tubes of different wall thicknesses.

the moving parts is $440 \mathrm{MPa}$; at $0.8 \mathrm{~mm}$, the maximum equivalent stress on the side wall of the moving parts is $560 \mathrm{MPa}$. By contrast, the metal tube with a thickness of $1.0 \mathrm{~mm}$ has a better energy absorption effect.

3.2.3. Comparison of Deceleration Performance of Moving Parts. The three displacement-time curves in Figure 8 show the movement of moving parts of metal tubes with different wall thicknesses when they are compressed. The three curves reach their peaks at three points $\mathrm{A}, \mathrm{B}$, and $\mathrm{C}$, respectively. This indicates that the thinner the metal tube, the slower the compression (albeit to a greater amount).

3.2.4. Summary of Results. The compression energy absorption performance parameters of the three kinds of metal tubes with different wall thicknesses are summarised in Table 3. The above analysis shows that the metal tubes with thicknesses of $1.2 \mathrm{~mm}$ and $1.0 \mathrm{~mm}$ have better energy absorption effects, making the equivalent stress of moving objects smaller, and the energy absorption performance of the two is similar.

3.3. The Influence of the Relative Position Relationship between the Metal Tube and the Compressed Body on the Compression Energy Absorption Performance. The energy absorption performance of the metal tube is studied in 
TABLE 3: Simulation results: compression and energy absorption parameters of metal tubes of different wall thicknesses.

\begin{tabular}{|c|c|c|c|}
\hline Wall thickness of the metal tube $D(\mathrm{~mm})$ & 1.2 & 1 & 0.8 \\
\hline Time of compression and energy absorption (ms) & 1.6 & 2 & 2.25 \\
\hline Biggest equivalent stress of the moving part ( $\mathrm{MPa})$ & 480 & 440 & 560 \\
\hline Energy absorption ratio of the metal tube (\%) & 93.8 & 93.5 & 87 \\
\hline
\end{tabular}

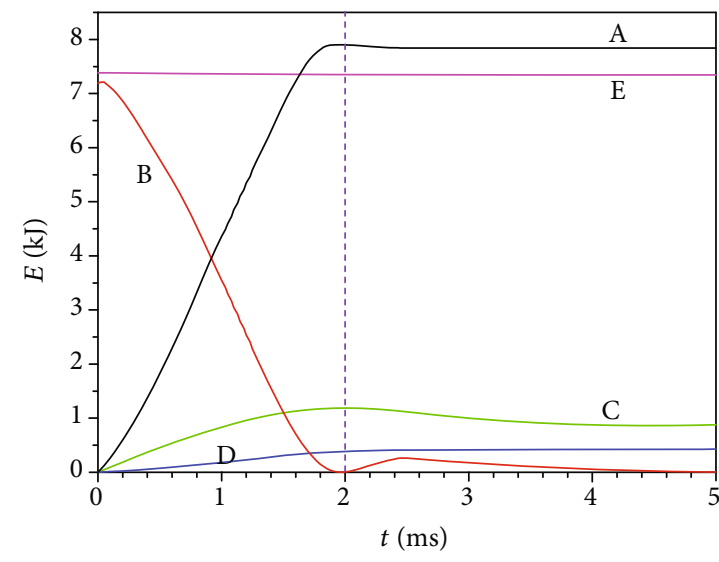

(a) $0.75 \mathrm{~mm}$

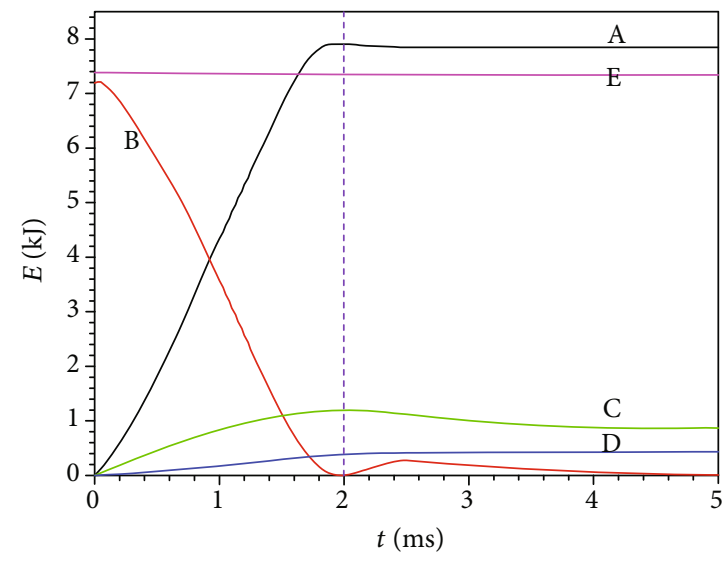

(b) $0.5 \mathrm{~mm}$

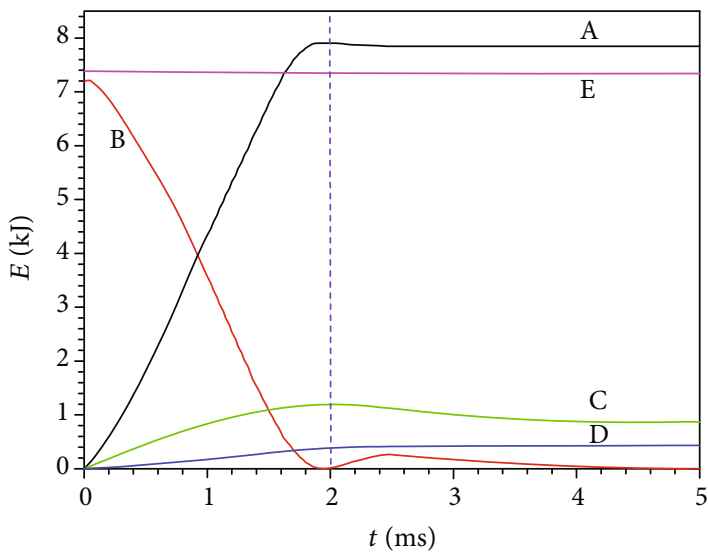

(c) $0.25 \mathrm{~mm}$

Figure 9: Energy conversion curves in the process of compression and energy absorption of metal tubes located in different positions. A: internal energy of the metal tube; B: kinetic energy of the ascending object; C: work done by external load; D: frictional energy consumption; E: total energy of the model.

different positions in the clearance of 1 and 2 moving parts. Using the metal tube with an outer diameter of $5 \mathrm{~mm}$ and a thickness of $1 \mathrm{~mm}$, making the distance from the outer wall of the metal tube to the wall of the inner moving object $0.75 \mathrm{~mm}$ (the metal tube is centred within the available clearance), $0.5 \mathrm{~mm}$ (the metal tube deviates from the centreline by $0.25 \mathrm{~mm}$ ), and $0.25 \mathrm{~mm}$ (the metal tube deviates from the centreline by $0.5 \mathrm{~mm}$ ), the buffer energy absorption over time is calculated.

3.3.1. Comparison of Energy Conversion Performance. Figure 9 shows the energy conversion in the process of simulation in different positions on the metal tubes. The energy absorption rate of the metal tube at $0.75 \mathrm{~mm}$ is $93.5 \%$. At $0.5 \mathrm{~mm}$, the energy absorption rate of the metal tube reaches $93.4 \%$, and at $0.25 \mathrm{~mm}$, the energy absorption rate of the metal tube is $92.2 \%$. Therefore, there is little difference in the energy absorption rate of metal tubes under the three working conditions analysed here.

3.3.2. Comparison of Structural Stress Distribution. The stress distribution in each of the three conditions is shown in Figure 10. The degree of compression of the metal tube is almost the same, but the equivalent stress distribution on the wall of the moving part varies as follows: at $0.75 \mathrm{~mm}$, the maximum equivalent stress on the wall is $456 \mathrm{MPa}$; at $0.5 \mathrm{~mm}$, the maximum equivalent stress on the wall is $450 \mathrm{MPa}$; and at $0.25 \mathrm{~mm}$, the maximum equivalent stress on the wall is $417 \mathrm{MPa}$.

\subsubsection{Comparison of Deceleration Performance of Moving} Parts. The three displacement-time curves in Figure 11 show the movement of the ascending part under the action of the 


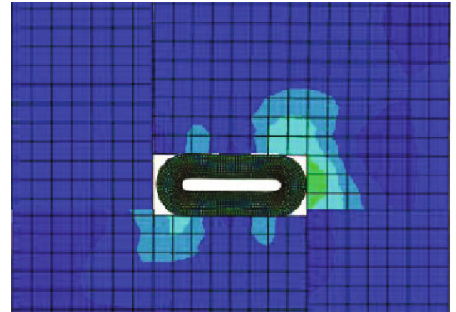

(a)

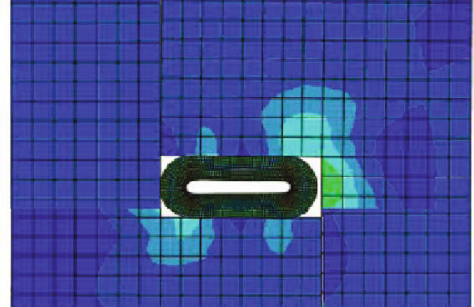

(b)

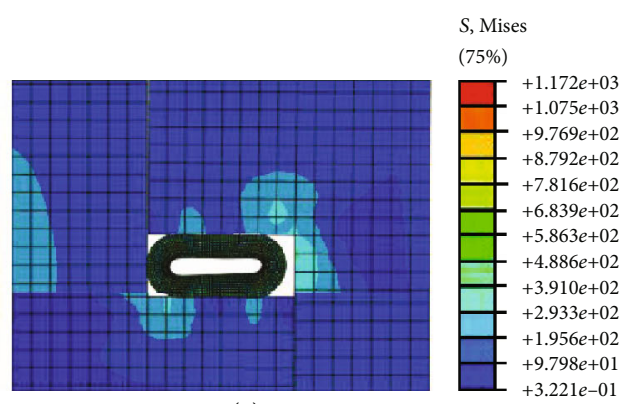

(c)

FIGURE 10: Compression equivalent stress for metal tubes displaced at different locations.

metal tube in different positions: the three curves are similar, and the peaks all lie near point $\mathrm{A}$, indicating that the motion of the ascending part is not the same in each case.

3.3.4. Summary of Results. Under the three working conditions in different positions, the compression and energy absorption performance parameters of the metal tube are listed in Table 4. From the above analysis, it can be seen that the energy absorption rate of thin-walled tubes under the three conditions varied little, but the stress on the wall is minimised at $0.25 \mathrm{~mm}$, indicating that the extrusion between the tube and the wall is minimised. Therefore, the metal tube has a better cushioning effect when it is close to the outer wall of the inner moving part.

3.4. The Influence of the Number of Stacked Metal Tubes on the Compression and Energy Absorption Performance. We chose a metal tube with an outer diameter of $5 \mathrm{~mm}$ and a wall thickness of $1 \mathrm{~mm}$, placed $0.25 \mathrm{~mm}$ from the outer wall of the ascending part. The numbers of superimposed tubes were set to 8,12 , and 16 , respectively. The process of buffering energy absorption was simulated, and the effects of different superimposed quantities on compression and energy absorption performance were compared.

3.4.1. Comparison of Energy Conversion Performance. The energy conversion obtained by simulation in different stacking numbers is shown in Figure 12: the energy absorption rate of 8 metal tubes is $84.9 \%$, the energy absorption rate of 12 metal tubes is $92.2 \%$, and the energy absorption rate of 16 metal tubes is $94.9 \%$. This shows that the energy absorption rate of the metal tube increases with the number of stacked tubes.

3.4.2. Comparison of Structural Stress Distribution. The finite element simulation results of different stacks of metal tubes which are compressed and collided are shown in Figure 13. It shows that the metal tube is compressed to the greatest extent when using 8 tubes (then 12 and 16, in descending order). The maximum stress on the wall of the moving parts is $520 \mathrm{MPa}$ (8 tubes), $417 \mathrm{MPa}(12$ tubes), and $351 \mathrm{MPa}$ (16 tubes). The more metal tubes are stacked, the lower the compression thereof and the lower the stress on the moving parts.

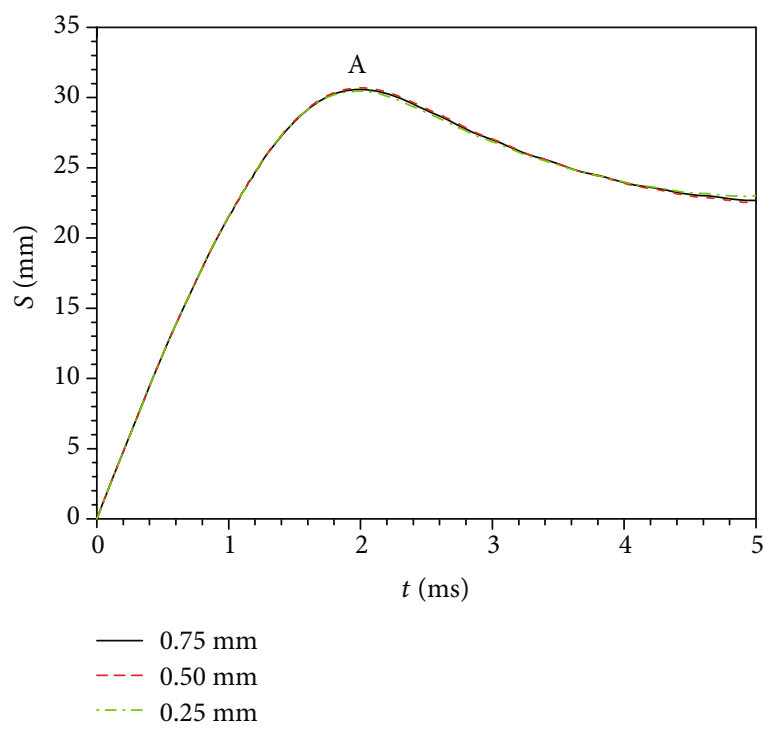

FIGURE 11: Displacement-time curves of the ascending object of metal tubes displaced at different places.

3.4.3. Comparison of Deceleration Performance of Moving Parts. The motion of the ascending part under the action of the metal tubes with different numbers of stacked tubes is shown in Figure 14: the more tubes in the stack, the greater the buffer energy absorption and travel and displacement time.

3.4.4. Summary of Results. The compression and energy absorption performance parameters of the metal tubes under the three different working conditions are listed in Table 5. With the increase in the number of the metal tubes, the buffer stroke and time increase, the tube compression decreases, the stress on the moving part decreases, and the energy absorption rate of the thinwalled tube increases; however, from an engineering viewpoint, fewer metal tubes should be selected to meet the needs of engineering practice.

\section{Experimental Verification}

Three dynamic compression tests were designed, and metal tubes of $5 \mathrm{~mm}$ in diameter and $1 \mathrm{~mm}$ in wall thickness were placed in the centre. The three working conditions are set 
TABLE 4: Simulation results of compression and energy absorption parameters of metal tubes located in different positions.

\begin{tabular}{|c|c|c|c|}
\hline Location of the metal tube $d(\mathrm{~mm})$ & 0.25 & 0.5 & 0.75 \\
\hline Time of compression and energy absorption (ms) & 2 & 2 & 2 \\
\hline Biggest equivalent stress of the moving part ( $\mathrm{MPa})$ & 417 & 450 & 456 \\
\hline Energy absorption ratio (\%) & 92.2 & 93.4 & 93.5 \\
\hline
\end{tabular}

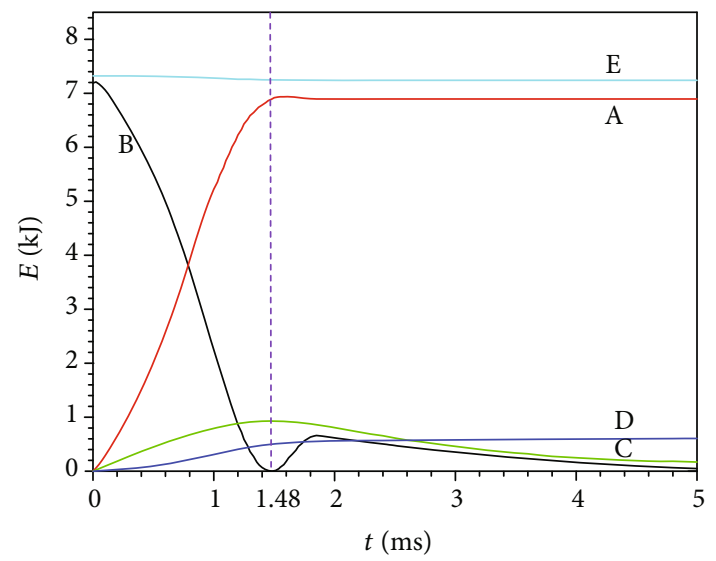

(a) 8 metal tubes

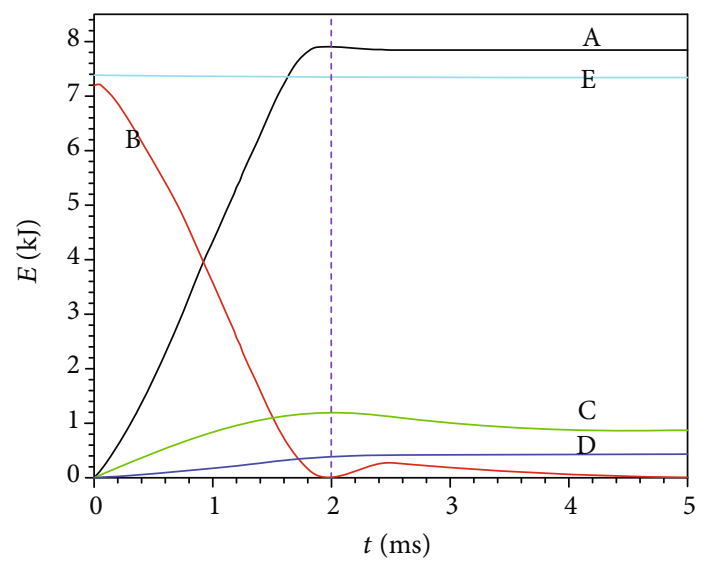

(b) 12 metal tubes

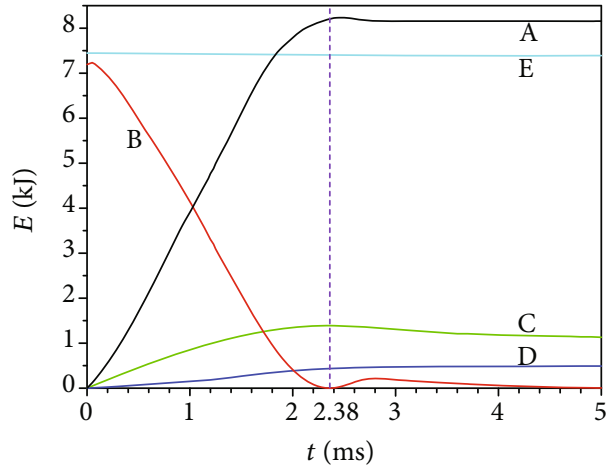

(c) 16 metal tubes

FIGURE 12: Energy conversion curves in the process of compression and energy absorption of different numbers of stacked tubes.

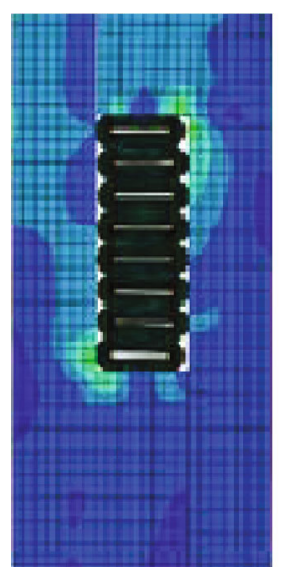

(a)

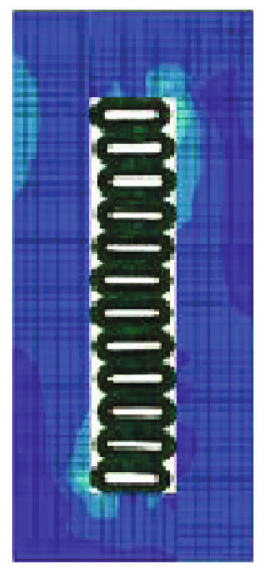

(b)
$S$, Mises

$(75 \%)$

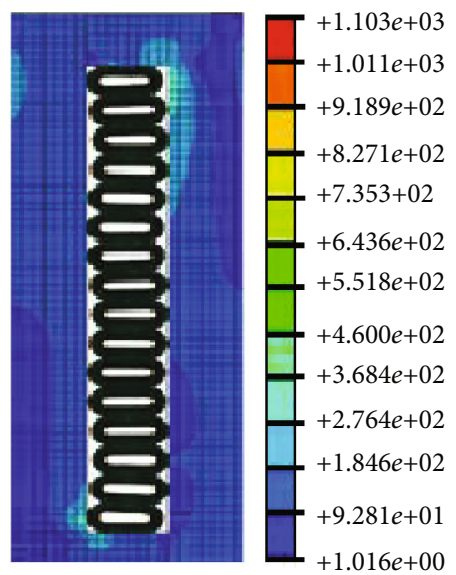

(c)

FIGURE 13: Compression of the metal tube stacks under collision (distribution cloud picture of equivalent stress). 


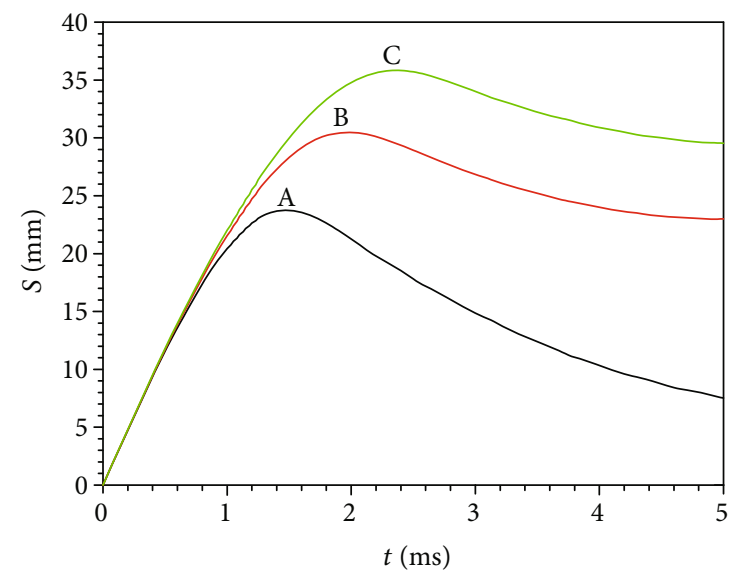

FIGURE 14: Displacement-time curves of the ascending object for stacked tubes. A: 8 metal tubes; B: 12 metal tubes; C: 16 metal tubes.

TABLE 5: Simulation results: energy absorption process parameters for different numbers of metal tubes.

\begin{tabular}{lccc}
\hline Number of metal tubes & 8 & 12 & 16 \\
Time of compression (ms) & 1.48 & 2 & 2.38 \\
Displacement of compression and energy absorption (mm) & 23.74 & 30.47 & 35.82 \\
Biggest equivalent stress on the wall of the moving part (MPa) & 520 & 417 & 351 \\
Energy absorption ratio of the thin-walled tube (\%) & 84.9 & 92.2 & 94.9 \\
\hline
\end{tabular}

TABLE 6: Comparison of simulation and test results.

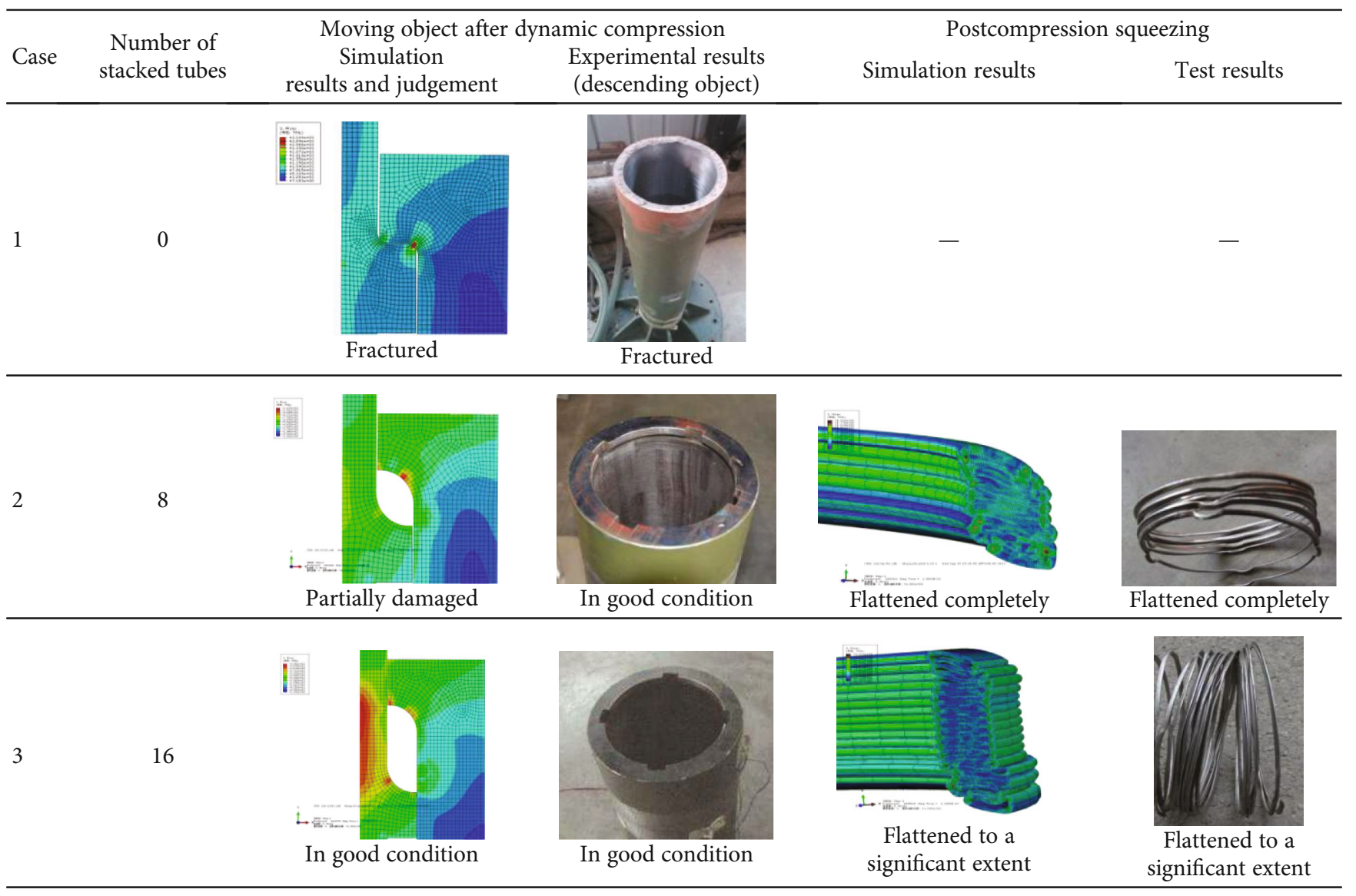


TABLE 7: Thickness of metal tubes under compression.

\begin{tabular}{lcccc}
\hline \multirow{2}{*}{ Case } & Number of stacked tubes & Simulation results & Total thickness of metal tubes as compressed (mm) \\
& & - & - & - \\
1 & 0 & 9.78 & 10.5 & \\
2 & 8 & 20.11 & 22.3 & $6.86 \%$ \\
3 & 16 & $9.82 \%$ \\
\hline
\end{tabular}

based on the numbers thereof in each stack (condition 1: no tubes, condition 2: 8 tubes, and condition 3: 16 tubes). The experimental results are listed in Table 6 and can be seen to be in agreement with the conclusions drawn from the simulation. As is shown in Table 6, the state of moving parts after dynamic compression and metal tube extrusion is as follows: stop-end face fracture occurs in descending parts without a metal tube (as seen in the simulation); with 16 stacked tubes, the ascending part and descending part are in good condition, and most of the metal tubes are compressed and flattened (as seen in the simulation); and with 8 stacked tubes, they are all flattened (as seen in the simulation).

The thickness of the compressed metal tube was measured and compared with the simulated results (Table 7). The results show that the thickness of the buffered tube in the experiment is slightly larger than that simulated (the discrepancy is within $10 \%$, suggesting that the model should be modified in future research).

\section{Conclusion and Discussion}

The energy absorption properties of hollow metal tubes in terms of diameter, wall thickness, and relative position were simulated. The behaviour in compression and energy absorption of various metal tubes was described.

(1) The compression and energy absorption properties of metal tubes are affected by their outer diameter, according to the placement space, and a matching outer diameter of a metal tube can be found to play a better effect on buffering and energy absorption

(2) The compressive and energy absorption properties of the metal tubes are affected by the wall thickness. When other conditions are the same, the smaller the wall thickness, the poorer the energy absorption ability of the metal tube, and the wall thickness increases and the energy absorption ability will increase, but when the wall thickness reaches a certain level, the increase in buffer and energy absorption decreases

(3) The buffering effect of the metal tube is related to its position within the gap. The closer the metal tube is to the inner compression body, the less damage it inflicts on the structural integrity of the outer compression body and the better the effect of the buffer and energy absorption, but the general influence is limited
(4) The greater the number of stacked metal tubes, the better the overall buffering and energy absorption properties; however, in practical application, it should be considered comprehensively from the space, weight, and stability of the metal tubes after stacking

(5) The simulation results and experiment results show that the small-sized metal tubes aligned so as to ameliorate the compaction of the compression process can have significant effects on the motion of bodies in a high-speed collision, and the buffer design needs to be aimed at matching the size and space requirements prevailing in practice

\section{Data Availability}

The test data used to support the findings of this study are included within the article.

\section{Conflicts of Interest}

The authors declared no potential conflicts of interest with respect to the research, authorship, and/or publication of the article.

\section{References}

[1] G. Li, "Development of strategic missile ejection technology," Missiles and Space Vehicles, vol. 7, pp. 38-39, 1990.

[2] A. Baroutaji, M. Sajjia, and A.-G. Olabi, "On the crashworthiness performance of thin-walled energy absorbers: recent advances and future developments," Thin-Walled Structures, vol. 118, pp. 137-163, 2017.

[3] C. Bisagni, "Crashworthiness of helicopter subfloor structures," International Journal of Impact Engineering, vol. 27, no. 10, pp. 1067-1082, 2002.

[4] J. Marsolek and H.-G. Reimerdes, "Energy absorption of metallic cylindrical shells with induced non-axisymmetric folding patterns," International Journal of Impact Engineering, vol. 30, no. 8-9, pp. 1209-1223, 2004.

[5] Z. Ahmad and D. P. Thambiratnam, "Application of foamfilled conical tubes in enhancing the crashworthiness performance of vehicle protective structures," International Journal of Crashworthiness, vol. 14, no. 4, pp. 349-363, 2009.

[6] A. G. Mamalis, M. Robinson, D. E. Manolakos, G. A. Demosthenous, M. B. Ioannidis, and J. Carruthers, "Crashworthy capability of composite material structures," Composite Structures, vol. 37, no. 2, pp. 109-134, 1997. 
[7] Z. Wang, X. Wang, C. Shi, Z. Li, and W. Zhou, "Mechanical behaviors of square metallic tube reinforced with rivets-Experiment and simulation," International Journal of Mechanical Sciences, vol. 163, article 105118, 2019.

[8] J. M. Alexander, "An approximate analysis of the collapse of thin cylindrical shells under axial loading," The Quarterly Journal of Mechanics and Applied Mathematics, vol. 13, no. 1, pp. 10-15, 1960.

[9] W. Abramowicz and N. Jones, "Dynamic progressive buckling of circular and square tubes," International Journal of Impact Engineering, vol. 4, no. 4, pp. 243-270, 1986.

[10] W. Abramowicz and N. Jones, "Dynamic axial crushing of square tubes," International Journal of Impact Engineering, vol. 2, no. 2, pp. 179-208, 1984.

[11] T. Wierzbicki, S. U. Bhat, W. Abramowicz, and D. Brodkin, "Alexander revisited-A two folding elements model of progressive crushing of tubes," International Journal of Solids and Structures, vol. 29, no. 24, pp. 3269-3288, 1992.

[12] R. Shams, A. Niknejad, A. G. Olabi, and M. Z. Nejad, "Quasi-static flattening energy absorption process on preformed circular tubes by numerical and experimental analyses," Thin-Walled Structures, vol. 144, article 106260, 2019.

[13] T. X. Yu and G. Lu, Energy Absorption of Materials and Structure, Chemical Industry Press, Beijing, China, 2006.

[14] S. Muthukumar and R. DesRoches, "A Hertz contact model with non-linear damping for pounding simulation," Earthquake Engineering \& Structural Dynamics, vol. 35, no. 7, pp. 811-828, 2006.

[15] A. A. Taflanidis, "Optimal probabilistic design of seismic dampers for the protection of isolated bridges against nearfault seismic excitations," Engineering Structures, vol. 33, no. 12, pp. 3496-3508, 2011.

[16] S. Liu, J. B. Liu, and E. Fang, "The advance of studies on the dynamic contact problem and its numerical methods," Engineering Mechanics, vol. 16, no. 6, pp. 14-28, 1999.

[17] N. Li and J. Li, "Numerical analysis of axial impact behavior of high strength steel thin-walled tube-beam structure [J]," Journal of Jiangsu University, vol. 31, no. 2, pp. 174-178, 2010.

[18] X. Wang, "MSC.DYTRAN instruction," Acta Armamentarii, vol. 21, no. S1, pp. 92-94, 2000. 